\title{
Geomatics Techniques for Submerged Heritage: a Mobile App for Tourism
}

\author{
VINCENZO BARRILE ${ }^{1}$, ANTONINO FOTIA ${ }^{1}$, ERNESTO BERNARDO ${ }^{1 *}$, GABRIELE \\ CANDELA $^{1}$ \\ ${ }^{1}$ Geomatics Lab, Department of civil engineering, energy, environment and materials (DICEAM) \\ Mediterranea University of Reggio Calabria \\ Via Graziella Feo di Vito, 25 - 89124 Reggio Calabria \\ ITALY
}

\begin{abstract}
The tourism sector is one of the major industries in Italy that has a high impact on local communities in terms of employment and economy. To show the results of a research project highlighting the use of new technology in the tourism sector, the Geomatics Laboratory of the Mediterranea University of Reggio Calabria has developed a mobile application for tourism. The case study is "Calarcheo Park", a nonaccessible underwater archaeological park located in Reggio Calabria, near the remains of the old walls of the ancient city of Reggio Calabria (Reghion, dating back to about the VIII B.C.). The main aim of this paper is to describe the process to relive and re-propose the experience of a real immersion through a journey in virtual reality. Particular attention was given to the research part concerning the simplification processes of the models inserted within an app for tourism sector. The concept app includes scenarios (seabed) and objects (Castle and artefact) obtained from three-dimensional models realized through photogrammetric techniques, therefore "heavy" models in terms of space necessary to store them, recall them and possibly process them. Considering the visualization purpose, and the metric precision needed, the $3 \mathrm{~d}$ models were reconstructed using a rapid method. To minimize the difficulties during the direct underwater survey carried out by a human operator (e.g. limited time available during the survey, accentuated inaccuracy due to human errors), the operations were carried out using an experimental ROV (Remote Operated Vehicles), widely used to explore underwater environments, equipped with cameras to perform photogrammetric acquisition in a single dive. To enhance the results and quality of the $3 \mathrm{~d}$ model, a procedure to improve the image quality, and optimizing the processing is also described. This work therefore illustrates the possibilities of using 3D models created by geomatics techniques within virtual environments for apps for tourism purposes.
\end{abstract}

Key-Words: - Remote Operated Vehicles, Virtual Reality, Underwater Photogrammetry, 3d model.

Received: March 3, 2020. Revised: July 13, 2020. Re-Revised: July 25,

2020. Accepted: July 28, 2020. Published: July 29, 2020.

\section{Introduction}

The Geomatics Laboratory of the Mediterranean University of Reggio Calabria has developed an app for tourism, to relive in a virtual immersive journey the experience of a real immersion using virtual reality.

The use of three-dimensional models obtained from photogrammetry within virtual reality and augmented reality apps requires high processing power and not always available in the most common devices. In this regard, an attempt was made to lighten the models obtained by reducing the number of edges and vertices that make up the structure without losing the geometric characteristics where required in terms of precision.

Unlike what is commonly used in the design of apps today, in this work we have tried to use 3D models obtained from photogrammetric processes as main scenes, instead of the images that create their outlines.

The development was carried out using Unity $3 d$ as multi-platform.

The scenarios and the objects presented in the scene (seabed and artefacts models) were obtained 
performing a three-dimensional photogrammetric survey; the seabed was detected using a submarine drone, while the models were obtained using a virtual simulated survey from Google Earth (allowing a strong simplification of the acquisition time, and minimizing risks related to survey performed by a technician).

The proposed mock-up of the application allows the user to enter into a scenario like that of virtual reality without being just mere spectators. The users become protagonists through direct interaction, directly participating in the immersion.

The main features of the mobile application can be summarized as:

a) Possibility to identify important archaeological sites nearby, using mobile device GPS coordinates; in this way it promotes and enhances the understanding of the context of archaeological sites, even in an underdeveloped area, improving the ability to attract visitors.

b) Allows to defend and enhance the cultural heritage, through the creation of $3 \mathrm{~d}$ models and digital archives that provide also sufficient information for a possible faithful reconstruction in case of destructive events.

c) The main scenes and 3D models inserted within the scenes of the app have been reconstructed from underwater images processed with innovative techniques that enhance their contours and shapes, allowing us to have excellent results in terms of quality.

\section{Materials and Methods}

The aim of this note is to present the processes that lead to the implementation of the app, from the survey to the construction of $3 \mathrm{D}$ models, to the assignment of augmented and virtual reality content, as well as to the user's Tracking system in the virtual environment.

The survey operations of the artifacts and the seabed were carried out through the use of a submarine ROV; the acquired images were improved for the subsequent three-dimensional reconstruction phase (clearly with regard to the seabed, the focus was more from a graphic than a metric point of view); instead, a speedy procedure was carried out with regard to the acquisition of images of the Aragonese castle and its 3D reproduction (useful only for graphic purposes). The models obtained were inserted and processed in the Unity environment, assigning the necessary scripts from time to time.

A key role was played by the lightening phase of the models in terms of "weight" (removal of edges and vertices) which allowed the app to be used without particular problems when loading the various scenes, given that the main scenes are formed from reconstructed and fully explorable 3D models.

\subsection{Case Study}

The survey activities have been carried out in different areas (Fig. 1) of the city of Reggio Calabria (south Italy), the results obtained were used to develop a tourist app to support, complete and repropose the touristic services of "Calarcheo" (a touristic submerged park) through virtual reality.

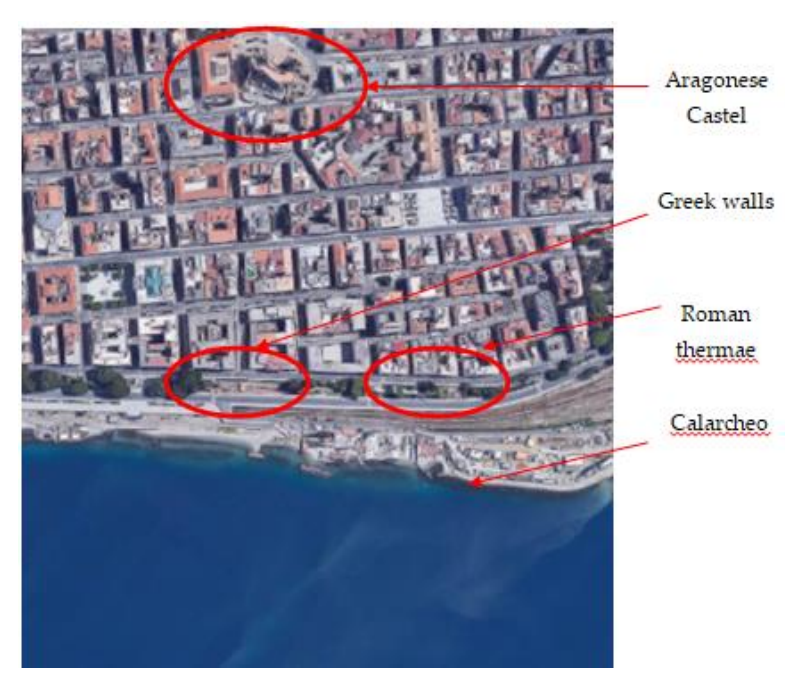

Fig. 1: Study Area.

"Calarcheo" is an underwater archaeological park located in Reggio Calabria, in the southern part of the "Lungomare Falcomatà", in a water surface of about $500 \mathrm{~m}^{2}$ from the shoreline and at a depth of about 40 meters. It is located near the Greek walls and Roman thermae (Fig. 2), near the old walls of Reghion (walls of the ancient city of Reggio Calabria, dating back to about the seventeenth century B.C.), and near to "Aragonese Castle". Today the archaeological site is not visitable because of the re-urbanization, for this reason, the goal of reliving in the augmented reality the experience was set.

The park also had as its purpose research and the following scientific activities: environmental and archaeological protection and enhancement, also for possible employment purposes; the dissemination of knowledge of the submerged archaeological heritage and the biology of marine environments; conducting educational programs for the improvement of culture in the field of Archaeology, marine biology and ecology; the realization of scientific programs to deepen the knowledge and the study of the area; the promotion of a compatible 
socio-economic development, favouring the traditional local activities already present, the resident citizens and the companies based in the municipalities falling within the area.

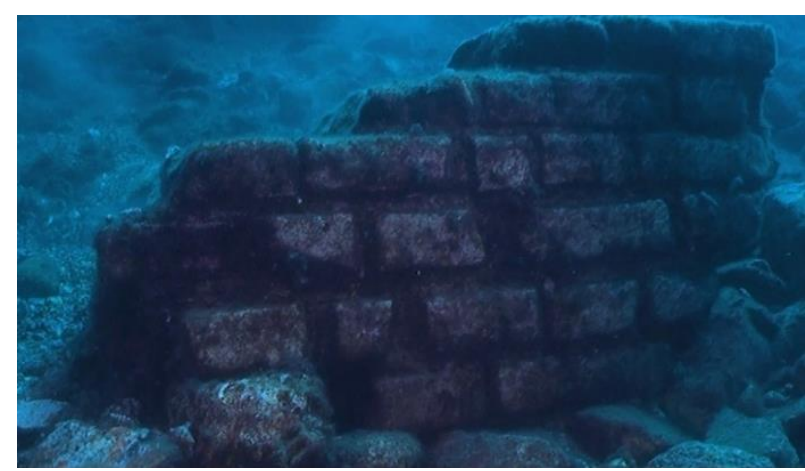

Fig. 2: Submerged Wall of Reghion.

In this regard, considering the common purpose, with a view to a desirable collaboration among the Geomatics Laboratory and the institutions that started the projects, we integrated the provided service of Calarcheo park with a virtual tour implemented into a touristic educational app.

Today it is possible to visit the original area's park only from the shore or boat dives. The mobile application allows the tourist to make a virtual immersion to re-enact the archaeological area through the new techniques of reconstruction of $3 \mathrm{D}$ scenarios, integrating the original contribution proposed by the park itself.

In fact, the app also allows those who cannot dive, to be able to admire what is present on the seabed directly on the device, thanks to the tracking phase that replaces the range of coordinates of the study area with fictitious coordinates within which the user is free to move.

Using mobile device position through GPS coordinates, when the user is within a range of the park, he will receive a notification to start the immersive tour.

The main archaeological artefact found in Calarcheo area seems to be an integral part of 'Porta Marina' and 'Fontana Nuova', that were the entrances through the old walls of Rhegion (Fig. 3).

The archaeological importance of the park and the area is now attested and confirmed also by the recent finds near the park.

The cultural heritage remains located on the seabed in the last decades of the 20th century have benefited from protection both nationally and internationally; preventive measures have been provided against the possibility of looting or destruction, as well as rules for in situ conservation of underwater cultural heritage as a priority option before authorizing or undertaking any intervention on it. Despite this, the activity of looting cultural heritage underwater is increasing rapidly.

The underwater cultural heritage is an invaluable testament to long-gone lifestyles. In most cases, these are testimonies of great historical and cultural significance.

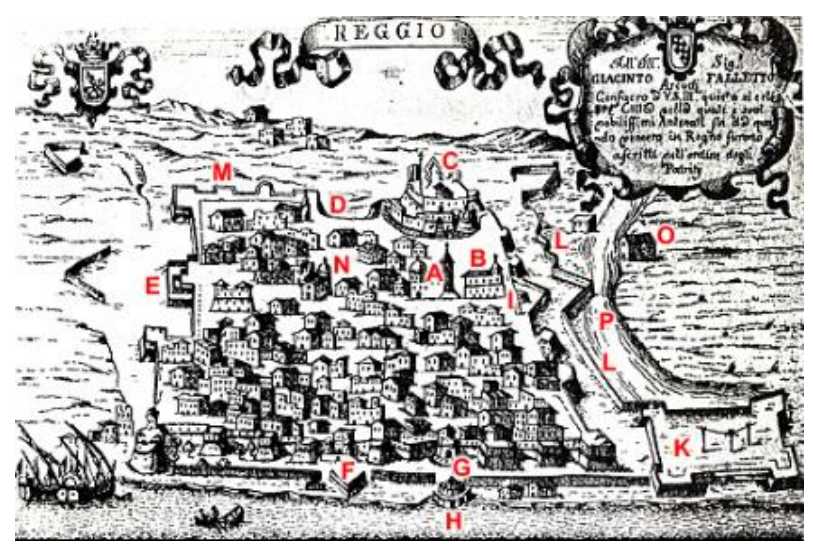

Fig. 3: Map of Reghion: C) Aragonese Caste; G) Porta Marina; H) Fontana Nuova.

Article 1 of the 2 November 2001 UNESCO Convention considers as a submerged cultural asset any trace of human existence of a cultural, historical or archaeological nature that has been partially or completely submerged, periodically or continuously for at least 100 years: sites, structures, buildings, artefacts and human remains, along with the archaeological or natural context in which they are located: ships, aircraft, other vehicles or any of them, their cargo or other content.

For this reason, a solution for virtual reality tour that concerned the review of the current testimony of the old walls of Rhegion was developed.

In the underwater archaeological park of Calarcheo, the path is marked by special white peaks that can be followed visually or tactilely. Along the submerged path different interesting areas can be found: remains of amphorae and relative shards, bases and columns of the old port of the marina dating back to the XVII century B.C., capitals, commonly called "cagnoli", which supported part of the arch of the door, a column not better dated.

\subsection{Submerged survey}

The seabed used within the main scene of the mobile app, is a backdrop detected through both an experimental [1] and a commercial ROV (Fig. 4), to test and verify its practicality and maneuverability. 


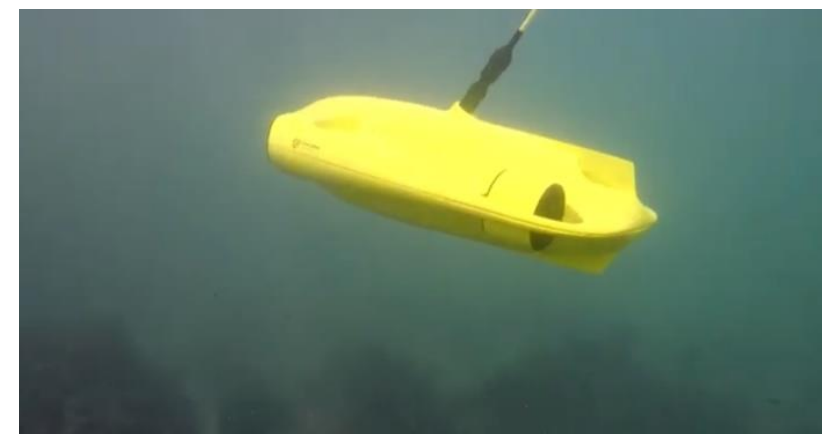

Fig. 4: Experimental ROV.

Thanks to the cable we can control the ROV from the shoreline or a boat, we can use the front camera and take photos, we can turn on the two led laterals and adjust their light intensity and at the same time be able to view the scene shot. The ROV has moved thanks to 2 main rear helipads that provide the driving force necessary for the navigation of about $4 \mathrm{knts}$, and 3 additional rotors arranged in triangles. A smart algorithm automatically controls and adjusts the setup with the Self Stabilizing Mode function and with the Depth Lock Mode function, automatically keeps depth constant. The same algorithm controls the angle of the hull that can be set by " $45^{\circ}$ " or $-45^{\circ}$ with the Adjustable Tilt Lock Mode function. The ROV has appeared extremely stable, thanks to the IMU (Inertial Measurement Unit) sensor, which works on 3 axes such as accelerometer, gyroscope and compass, and easily manoeuvrable. They may also experience other obstacles on the route, which could be avoided automatically. The camera mounted on the ROV has a CMOS sensor of $1 / 2.3$ with $12 \mathrm{MP}$ resolution and an optic with a $4 \mathrm{~mm}$ f.3.0 focal equivalent. The ISO sensitivity ranges from 100 to 3200 . Videos can be acquired at different resolutions: 4K or UHD (38402160 ) to $30 \mathrm{fps}$, or HD at $1920+1080$ to $30 \mathrm{fps}, 60 \mathrm{fps}$ and $120 \mathrm{fps}$. However, the quality of underwater images is strictly dependent on the water's clarity. So, the sunlight that penetrates from the air-water interface into the water is altered: more depth increases, more the lower temperature colours disappear. Moreover, the effect of refraction (depending on a number of parameters such as depth, temperature and salinity) can cause unstable modelling effects, so the intrinsic parameters of a submerged camera change significantly in comparison to the primary values. Suspension, little light at the high depths, affect the result exponentially and it is gradually necessary to illuminate the scene with LEDs.

\subsubsection{Image enhancement and 3d elaboration}

Depth, weather conditions, sea surface conditions deeply affect underwater colours. Furthermore, the effect of refraction generates unstable effects that disturb the modelling phase; so that, the intrinsic parameters of the camera change significantly concerning the primary values. The sunlight that penetrates the water is altered by different causes and effects, consequently, the colours are altered.

The scene is displayed as flattened, unbalanced and with little contrast. Increasing the distance between the object to be detected generates an increase in the altering effects [2]. For this reason, all applications that use altered images (such as underwater ones) require a prior colour correction phase, especially for the photogrammetric reconstruction. Two trends for image contrast enhancement (CE) are considered: direct, based on the improvement of a contrast measure or indirect, that operates on the image histogram, varying the intensity of the grey levels. Anyway, both procedures conduct to a stretching of the distribution of the grey levels requiring the tuning of features extraction procedure automatically. In this context, it is imperative the implementation of adaptive items manipulating data uncertainty. Different algorithms to enhance image has been used [3]. In this work to improve contrast and sharpness of the seabed's images, method A was applied: using the automated algorithms "Find Dark and Light Colours", Snap Neutral Midtones [4], [5], and the algorithm proposed by [6], changing the chromatic components by moving their distributions around the white point (white balance), improving the image contrast through the stretch of the luminance component. The first algorithm analyses the image to find dark and light colours and uses them as shadow and highlight colours. The second one allows the mid-tones to be adjusted so that the colours close to the neutral are mapped to the destination neutral colour. The results of the proposed methodology applied to the seabeds images are shown in Fig. 5-6.

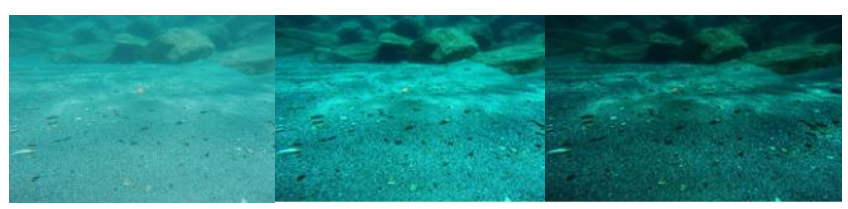

(a)

(b)

(c)

Fig. 5: Seabed image enhancements: (a) Before processing; (b) After sequence processing (c); After fuzzy processing. 
We observed that, as the depth increases and therefore light decreases the used procedure produces less precise results and the delimitation of the contours of objects has become increasingly difficult.

A new approach - Method B - was then used for image contrast enhancement based on fuzzy geometrical procedure; this adaptive procedure is based on a particular fuzzy formulation assisted by ascending order statistics and entropic evaluations [7]. In this context, Contrast Enhancement procedure can be thought as a transformation where the dark pixel must become darker and the light one must lighter; while the grey one must not change. Where dark, light and colours are instances of fuzzy quantities. The methodology originally used and optimized for black and white images has also been tested on the collected underwater dataset.

The following consideration on the images after processing by the designed procedure and histogram equalization can be made: in the images with strong bright illumination, architectural details have been preserved at the expense of sharpness. The processing by the designed procedure produces a very good level of $\mathrm{CE}$ even if the original darkness remains in the low right corner (Fig. 5) with welldefined edges.

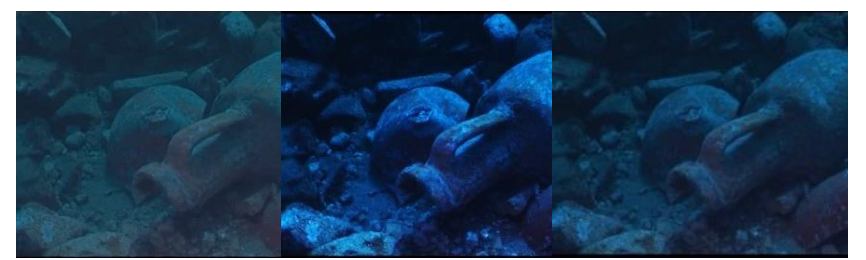

(a)

(b)

(c)

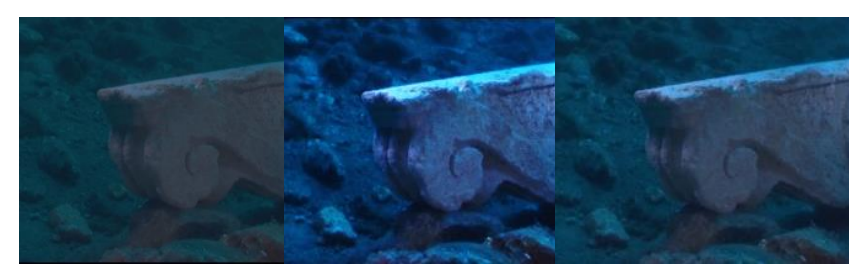

(a)

(b)

(c)

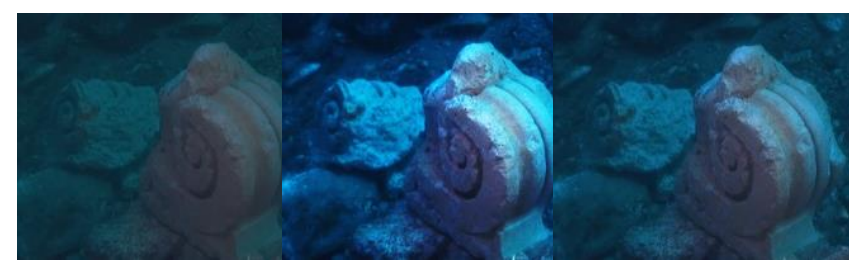

(a)

(b)

(c)

Fig. 6: Artefact 3 image by ROV: (a) After processing; (b) After sequence processing; (c) After fuzzy processing.
Although. in some cases, the second method presented turns out to be better (albeit slightly) for the processing of the artefact, it should be emphasized that the computational time required for processing does not justify the choice of the procedure used (it varies from the size and shape of the artefact and on average can be found in an increase of $60-65 \%$ ).

The enhanced images were processed using SFMMSV algorithms with commercial software Metashape (Agisoft, Russia), both for the realization of the 3D model of the seabed (Fig. 7) and of the artefacts (Fig. 8). Blurred or poor images in content (e.g. a homogeneous background) can worsen the results in the alignment step. For this reason, we set the calibration before the image's alignment phase. In this case, self-calibration was used. Camera information (sensor size and focal length) were automatically extracted from the EXIF file from the software.

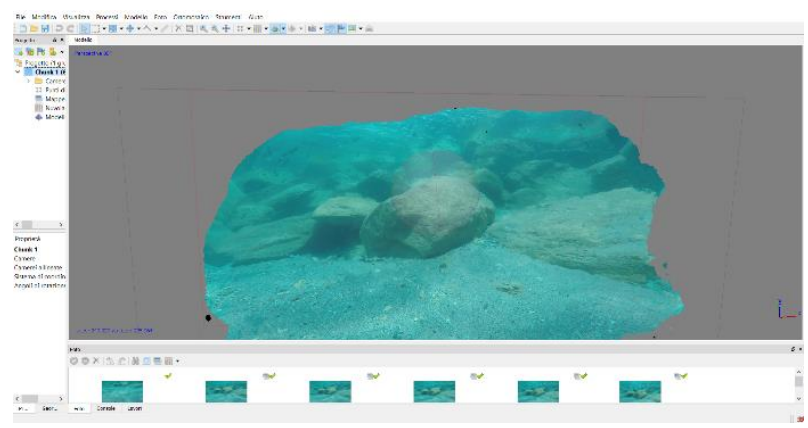

Fig. 7: Part of 3D modeling of a seabed.

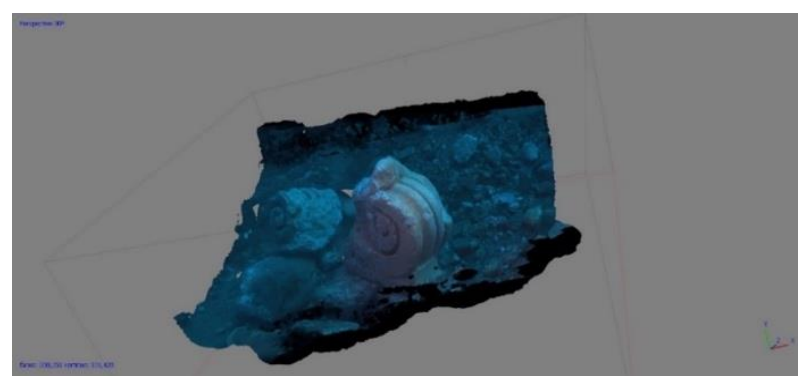

a)

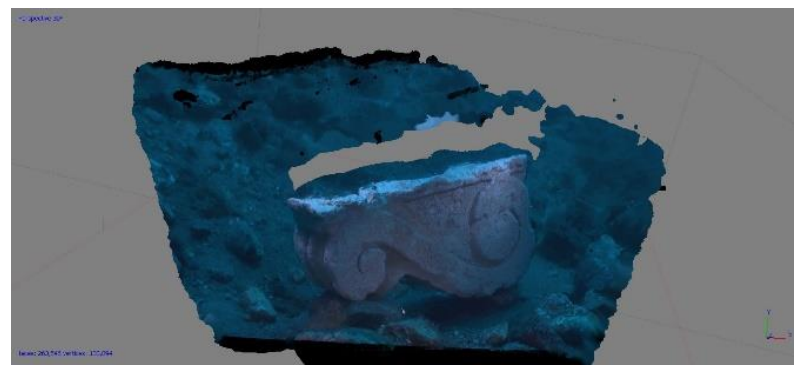

b) 


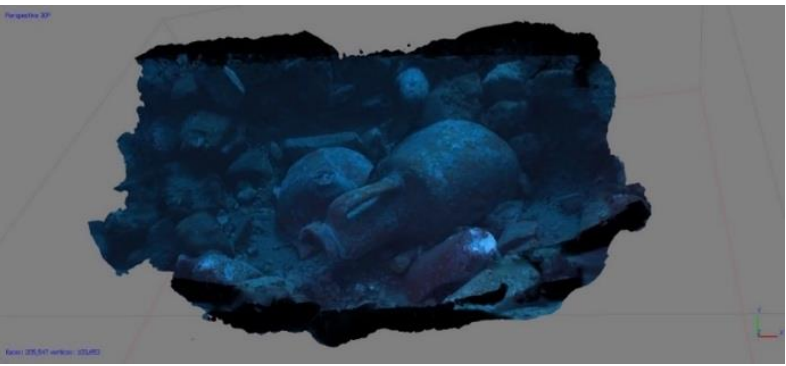

c)

Fig. 8: 3D model of: a) Artefact 1; b) Artefact 2; c) Artefact 3.

In 3D processing of the seabed, where it is not strictly necessary to appreciate the quality (even metric of the particulars), we use images elaborated with the first method, while the images of the detected artefacts need further processing to have 3D models of appreciable quality (B method).

\subsection{Data acquisition: Aragonese Castle}

As known, the methodologies for the realization of 3D models are manifold and each one presents different accuracy level of details, depending on the instrumentation and the methodology used related to the final use to which the work is destined.

In some parts of this case study, the metric accuracy of the $3 \mathrm{~d}$ model assumes a low importance cause to the didactic nature and the touristic finality of the application.

For this reason, we decided to carry out the 3D survey of the Aragonese castle, in a "fast way", using directly a dataset obtainable from Google Earth and then processed by classical photogrammetric techniques.

We used the Google Earth Pro program, setting the view on the castle at a $45^{\circ}$ angle. Subsequently, we created a tour, completing a $360^{\circ}$ turn around the building and maintaining the same angle of view, thus simulating the flight of a drone.

Later, through Movie Maker (in Google Earth Pro) we extracted images from the video with the following parameters: JPEG format (.jpg), size 1920 $\mathrm{x} 1080$ pixels (HD), 3 frames per second, maximum quality, obtaining about 86 images (Fig. 9).

We then proceeded with the automated photogrammetric processing program, (Agisoft Photoscan) to obtain a three-dimensional model with an acceptable definition mesh texture (Fig. 10). The workflow is automated from orientation of the images and for the generation to the model reconstruction. This condition led to an optimization of processing times ensuring good performance of the machine/software complex.

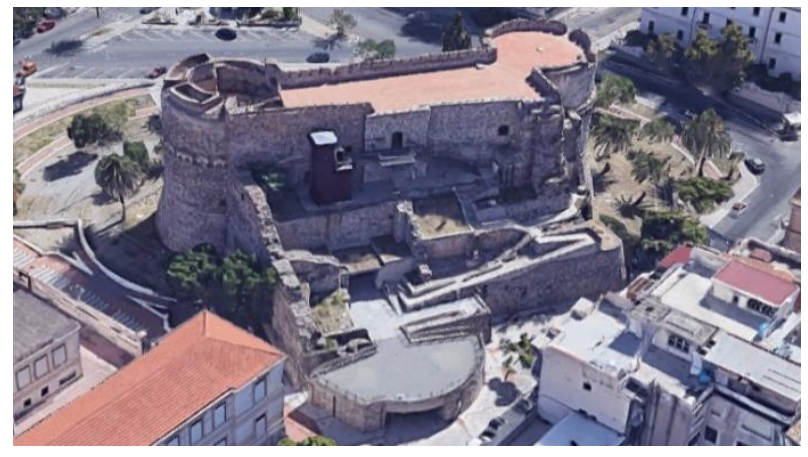

Fig. 9: Frame of Video.

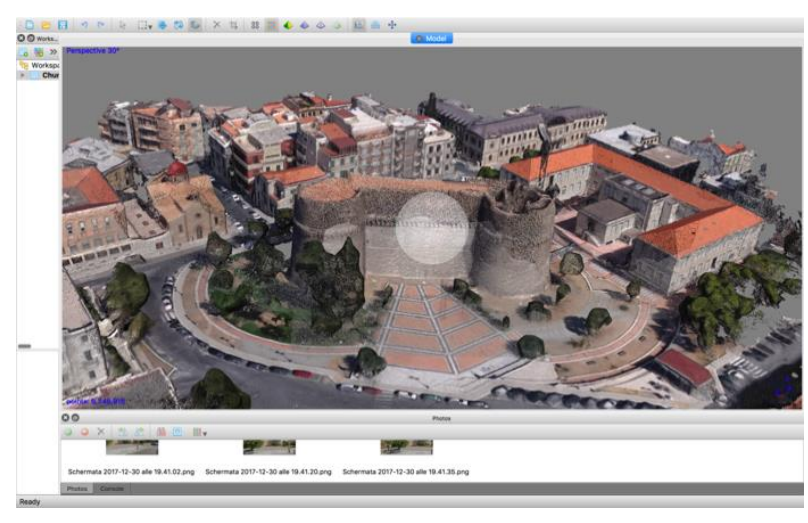

Fig. 10: 3D Texture.

To verify the level of accuracy achieved with this methodology, the result obtained by processing was compared both with the result obtained by a survey carried out by UAV (red line) and with that obtained by laser scanner (blue line). While the precision between the two rigorous procedures are almost equal, as we expect, the precision between the model generated by this application presents an accuracy lower than $3 \mathrm{~d}$ model generated with a laser scanner or UAV (Fig. 11).

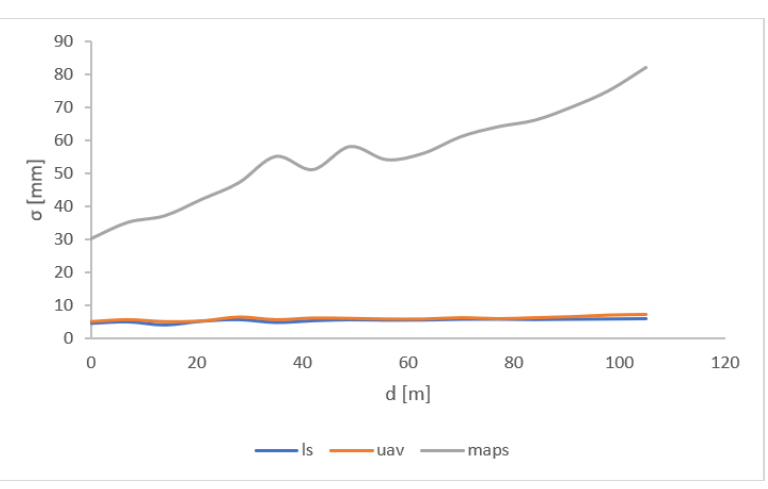

Fig. 11: Comparison among Laser scanner, Photogrammetry from UAV and Virtual Photogrammetry from Google Earth. 
As mentioned, the 3D model virtual photogrammetry was therefore used which guarantees, for the purposes of the proposed app, a good graphic representation of the object that we want to contextualize within the app

\section{Mobile application for immersive tour in virtual reality}

The tourism sector is one of the biggest industries in Italy relevant for the local communities economically and in terms of employment.

In Italy, the figures record positive numbers, with tourist flows (in search of natural, cultural, historical, artistic and food and wine experiences) increasing throughout the Peninsula.

A trend that seems destined to increase even in the following years.

Therefore, in a market rich in offerings (our country is full of archaeological and cultural sites), we believe it is important to find ways to offer a different and new tourist experience to increase the appeal towards lesser-known archaeological sites [8; 9].

The Geomatics Laboratory of the Mediterranea University of Reggio Calabria has developed for research purpose a mobile application to show the potential of virtual reality for tourism.

The mock-up allows the user to visualize information (e.g. historical, multimedia) and interact through in the VR. It also makes it possible to perform a virtual tour with the 3D backdrop model as the main scene and to interact with the surrounding environment [10].

The main scene of the app is the 3D model of the seabed, however, background images have also been used to cover those parts that cannot be covered by the 3D model.

Therefore "real-time" refers to the user's ability to live a virtual tour while the area is framed or while the user is in the predefined coordinate range of the study area [10].

As long as we have a few objects and a few details in the scene, rendering will be very fast and the app will render a high number of frames per second [11].

However, when our scene contains many objects, we will notice a greater effort and a probable "lag", due to the rendering scene [12], so we can easily guess that, the greater the number of objects, the greater the waiting time.

The word objects, however, in this case, is incorrect, we should talk about triangles, that is the more triangles in a scene, the longer the rendering times know. As a result, the more details we will have in a scene and the more difficult it will be to be able to move it quickly in real-time in an app.

We can immediately understand that avoiding scenes with too many triangles is the basis of creating scenes intended for Realtime, [13-16].

To achieve this, the command decimated outside the "Edit Mode", with intensive values at 0.2, corrected the presence of the too irregular distribution of polygons using the "Remesh" function.

Finally, the intersection of objects was deleted through the "Boolean" function.

Precisely for this reason, it is preferable to use for applications of this type three-dimensional model obtained with methodologies that are less accurate in metric terms, they will certainly be preferable when simplifying the model.

Then having the $3 \mathrm{~d}$ model the user finds it as the background of the main scene and then can make all the features of the app.

In fact, through the tracking process, we built a correlation between the real coordinates detected through the device GPS and the coordinates of the georeferenced 3D model.

The process of iteration of real/virtual coordinates means that, when the coordinates of the user position detected by the GPS of the mobile fall within a pre-established range in the construction phase of the app, it starts a simulation of the exploration of the 3D model [17].

At this point it will be understood how the virtual tour is conceived as a sequence of scenes presented in succession over time, creating what is the sensation of movement.

Motion tracking, allows us to activate animations or videos based on the position and orientation of the device, move around an object and interact with it as if it were part of the environment and allows also to track the position of the device within the surrounding environment.

This type of tracking has already been implemented in the mobile app, as it allows the user to let the app know movements and react accordingly; the use of ARCore, a software development kit made by Google that is implemented within the Unity environment was used to obtain the desired result. ARCore receives information from the room and from the inertial sensors present in the hardware, on which the app run and through a process called Concurrent Odometry and Mapping (COM) determine the position of the smartphone that moves in space and correctly render the three-dimensional model in AR, looking realistic.

The software autonomously defining plans and feature points to identify the appropriate constraints 
for the correct positioning of the object in space and concerning to the camera.

In essence, it allows the smartphone to understand the scene, and once the location of the device is known, ARCore can position the objects in such a way that their interaction with the environment seems realistic.

It is also able to observe the surrounding light and illuminate objects as if they were part of the environment.

We performed the test on Unity 2019.2.13 and with ARCore SDK for Unity 1.13. After that we imported the three-dimensional model inside the scene and defined the laying position (Center Eye), two components were added to the scene: Track Pose Driver and ARCore Session; finally the Background was defined as ARBacground to see the object inside the device as if interacting with the surrounding environment.

The main scene is created starting from the $3 \mathrm{D}$ model with the texture of the area under examination. Points of interest (POIs) have been identified in it and multimedia data were assigned to each POI (audio file, a hypothetical 3D reconstruction of the Aragonese Castle and the walls, of additional information) (Fig. 12 - 13 - 14).

Users, when interacting with POIs, can both visualize information or move between tour scenes, thanks to the associated scripts [18].

The scripts associated are extensions of the base class "MonoBehaviour", that allows defining their actions thanks to the use of particular functions called "event handlers".

These procedures are chosen by the programmer that specify how to interact with the element, for example, in the event of a collision with another object (OnCollisionEnter) or at the beginning of the object's life cycle (Start).

The tracking processes (user trace in the model) and the registration (overlapping of virtual content) exploited both the presence of marker (such as the logo of the municipality or the entrance sign to the castle) and both the connection between the realtime coordinates detected through the device GPS and the model's coordinates.

The link between real coordinates and virtual coordinates means, for example, that once the app is launched, whenever the user is within a predetermined range or the identified area, it will be informed immediately, with the notification that the user can make the virtual tour [19].

In the display phase, due to the axes orientation changes from device to device, a control has been inserted into the code on the type of device used, which allows the realignment of the axes to obtain a scene display consistent with the reality [20].

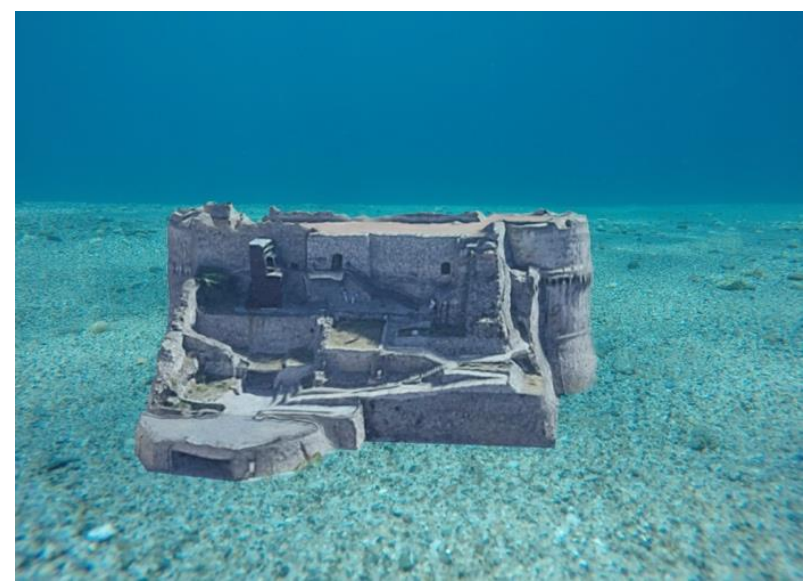

Fig. 12: Screen App: Aragonese Caste in Calarcheo seabed

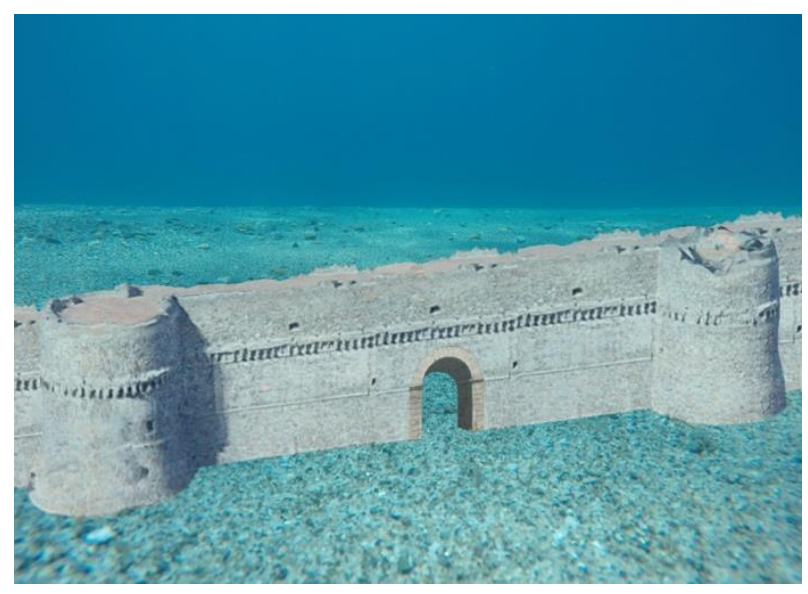

Fig. 13: Screen App reconstruction of Fontana Nuova and Porta Marina.

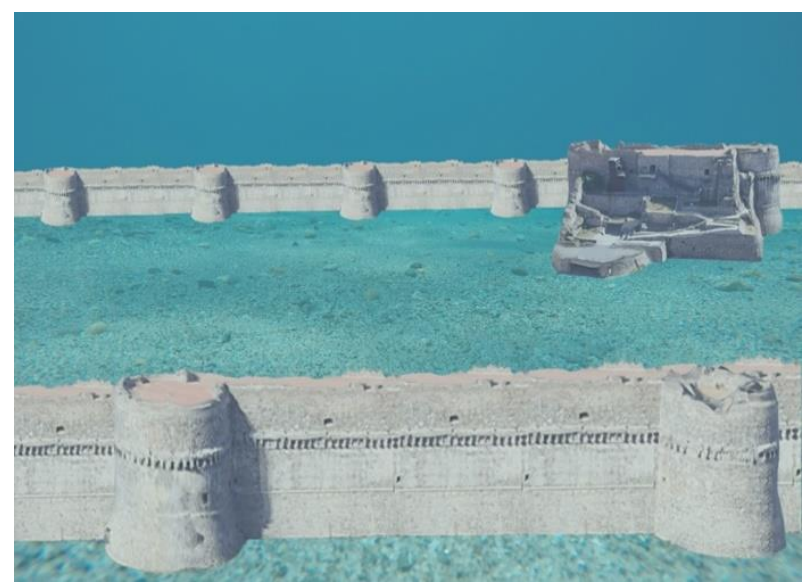

(a) 


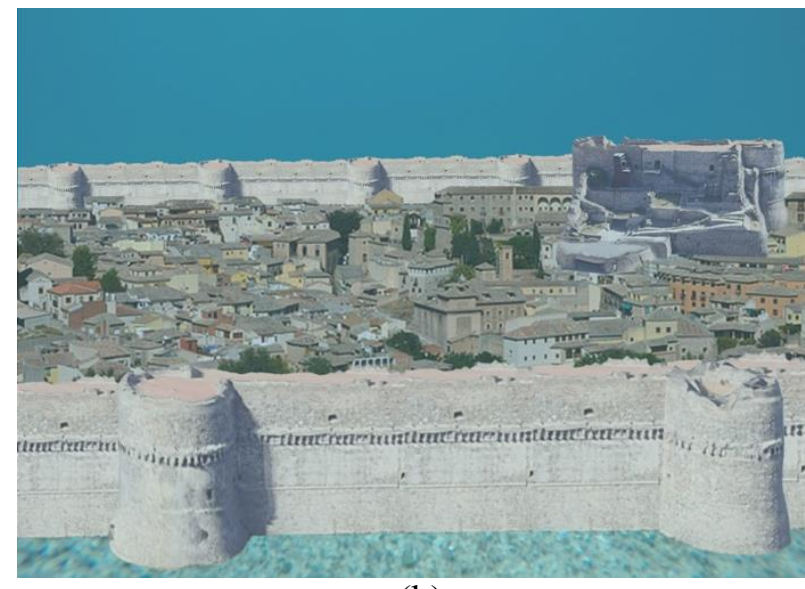

(b)

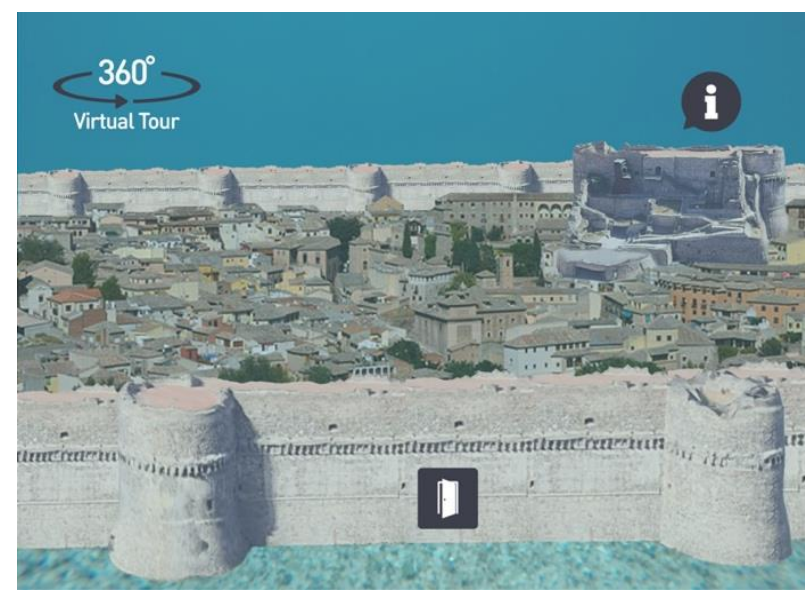

(c)

Fig. 14: Screen App

\section{Discussion}

Through an interdisciplinary dialogue between traditional geomatic techniques and new technologies in the virtualisation sector, we can improve the documentation and provide a useful tool for greater understanding, enhancement and conservation of the cultural heritage.

Today the use of smartphones is widespread. The creation of apps based on the techniques of virtual and augmented reality allows users to live special experiences. Moreover, the use of virtual and AR applications allows the user to see associated multimedia contents and information directly on the subject of investigation [21-25].

Unlike other apps of the same type in which the main scene is generally built through the only use of images, in this case, we would directly insert a 3D model to make it explorable. Besides, special imaging techniques were used in the application that enhances their contours and shapes, allowing us to have excellent results in terms of quality to improve the quality and precision of the 3D models and consequently the main scene.

Different from what was expected, it has been noted that it is not recommended to always use the same methodology, because we observed that while the depth increases and therefore the light decreases, this procedure produces less and less precise results and the delimitation of the contours of objects has become increasingly difficult.

For this reason, two different methods, one for the reconstruction of the seabed and the second for objects were used.

In the first methods the first algorithm analyses the image to find dark and light colours and uses them as shadow and highlight colours, and the second one allows the mid-tones to be adjusted so that the colours close to the neutral are mapped. The second method used to enhance the image contrast was based on fuzzy geometrical procedure; this is a new adaptive procedure based on a particular fuzzy formulation assisted by ascending order statistics and entropic evaluations - method B. The treatment by the designed procedure produces a very good level of CE even if the original darkness remains in the low right corner with well-defined edges. The images after treatment by the designed procedure and subsequentially histogram equalization can be considered good quality. Specifically, in the images with a strong bright, architectural details have been preserved at the expense of sharpness. As expected and verified, the precisions held with other important modes, are not comparable with those obtained with the methodology we choose for 3D modelling.

Nonetheless, precision is balanced by greatly reducing the heaviness of the $3 \mathrm{D}$ model and thus increase the load speed of the app. In this way, in applications of this type, the virtual survey methodology is recommended, using directly a dataset obtainable from Google Earth.

In the case study, the obtained result confirms the possibility to speed up the process of navigation between the app scene but giving the user the possibility to focalize on one specific object.

Furthermore, the use of specific models of "heavy" geomatics from the point of view of storage and real-time processing therefore makes the app in question particular of its kind. In fact, on the models of the artifacts where we wanted to coexist models which are not only well representative but also metric, we worked on their simplification (reduction of angles and edges and consequently also on the "weight") without changing their precision. On the other hand, an attempt was made to have a good 
graphic approximation of the models reconstructed expeditiously. The results obtained clearly can be improved from the point of view of the weight of the app, however it should be emphasized that navigating within the same scene did not generate loading problems. On the other hand, there are also noticeable slowdowns in the passage between scenes that foresee $3 \mathrm{D}$ models such as navigation environments.

The intertwining of the contents between them, through continuous intertextual and multimedia connections, allows the user to develop their own path in complete freedom.

Our work has attempted to illustrate that the use of digital data sets and immersive data tools in archeology requires new methodologies for archiving data collected in the field. However, we believe that our methodologies have proved feasible to display, analyze and cure three-dimensional stratigraphic data in an interactive virtual environment.

\section{Conclusion}

Development in the fields of the Underwater Cultural Heritage and maritime archaeology offers an interdisciplinary and creative academic framework.

The expansion of information and communication technologies (ICT) has been shown to offer great possibilities in the field of culture by developing new ways to improve the visitor experience in cultural interesting sites, changing the way as people perceive the environment around them.

The digital representation of real spaces provides users with the illusion of real presence within the study area. To date, Calarcheo Park visitors can access the site, directly from the beach or diving from a boat. Thanks to the development of the app and the use of new Virtual Reality and Augmented reality technologies, the park will be made accessible even without making immersion in the literal sense of the term, offering participants the opportunity to "digitally" visit places that are normally inaccessible

The benefits of such experiences can be defined in their ability to create meaningful connections between the real world and virtual visitors, while also allowing participants to interpret objects and environmental qualities on their own.

One of the main problems that the realized app showed (besides the presence of a very high number of edges and vertices) was the size, in fact even if we proceeded to simplify models to use them as main scene the size is not optimized. Developing the app itself in a progressive web application (PWA) could be a great way to address this problem. We refer to a new structure or technology.

\section{Acknowledgements:}

The authors thank Mr. Filippo Mallamaci, director of Scuba point association and responsible of Calarcheo Park, for the cooperation during field tests.

\section{References:}

[1] Barrile, V., Fotia, A., Ponterio, R., and Aliotta, F., Photogrammetric techniques for the reconstruction of underwater $3 \mathrm{~d}$ models of seabed and artifacts, Int. Arch. Photogramm. Remote Sens. \& Spatial Inf. Sci., Vol.XLII2/W10, 2019, pp. 25-30.

https://dx.doi.org/10.5194/isprs-archives-XLII2-W10-25-2019

[2] Bruno, F., Lagudi, A., Gallo, A., Muzzupappa, M., Petriaggi, B. D., Passaro, S., 3D documentation of archeological remains in the underwater Park of Baiae, International Archives of the Photogrammetry, Remote Sensing and Spatial Information Sciences ISPRS Archives, Vol.40-5/W5, 2015, pp. 4146.

https://doi.org/10.5194/isprsarchives-XL-5W5-41-2015

[3] Mangeruga, M., Cozza, M., Bruno, F., Evaluation of Underwater Image Enhancement Algorithms under Different Environmental Conditions, Journal of Marine Science and Engineering, Vol.6, Issue 1, 2018. https://doi.org/10.3390/jmse6010010

[4] Mangeruga, M., Bruno, F., Cozza, F., Agrafiotis, P., Skarlatos, D. Guidelines for Underwater Image Enhancement Based on Benchmarking of Different Methods. Remote Sensing, 10(10), 1652, 2018. https://doi.org/10.3390/rs10101652.

[5] Barrile, V., Fotia, A., and Bernardo, E., The submerged heritage: a virtual journey in our seabed, In. Arch. Photogramm. Remote Sens. Spatial Inf. Sci., Vol.XLII-2/W10, 2019, pp. 17-24.

https://doi.org/10.5194/isprs-archives-XLII-2W10-17-2019

[6] Bianco, G., Muzzupappa, M., Bruno, F., Garcia, R., Neumann, L., A new color correction method for underwater imaging. Arch. Photogramm. Remote Sens. Spat. Inf. Sci., Vol.XL-5/W5, 2015, pp. 25-32. 
10.5194/isprsarchives-XL-5-W5- 25-2015

[7] Versaci, M., Morabito, F. C. and Angiulli, G., Adaptive Image Contrast Enhancement by Computing Distances into a 4-Dimensional Fuzzy Unit Hypercube, IEEE Access, VOL.5, 2017, pp. 26922-26931.

[8] Brumana, R., Tucci, G., Lerma, J.L., Special Section Preface: Informative Models and Systems for Virtual Museum. Virtual Archaeology Review, [S.l.], VOL.10, No.21, 2019, ISSN 1989-9947.

https://doi.org/10.4995/var.2019.12357.

[9] Lerma García, J. L., Cabrelles Lopez, M., Navarro Tarin, S., Galcera Ustero, S. Documentación 3D y visualización multimedia de la Cova del Parpalló (Gandia). Virtual Archaeology Review, [S.l.], VOL.1, No.2, 2010, pp. 123-127, may. ISSN 1989-9947. doi: https://doi.org/10.4995/var.2010.4701.

[10] Hellman, T., Lahti, M., Photogrammetric 3D Modelling for Virtual Reality, WindCoE, 2018. https://www.researchgate.net/project/WindCoE $-3$

[11] Botsch, M., Kobbelt, L., Pauly, M., Alliez, P., Lévy, B., Polygon Mesh Processing, Taylor \& Francis Inc, 2010.

[12] Chopine, A., 3D art essentials. The fundamentals of $3 D$ modelling, texturing, and animation, Burlington: Focal Press, 2011.

[13] Graham, K., Fai, S., Dhanda, A., Smith, L., Tousant, K., Wang, E., Weigert, A., The VR Kiosk. In Ioannides M. (eds) Digital Cultural Heritage. Lecture Notes in Computer Science, . Springer, Cham, Vol.10605, 2018, pp. 324336.

[14] Stankovic, S., Virtual Reality and Virtual Environments in 10 Lectures. Morgan \& Claypool, 2016.

https://doi.org/10.2200/S00671ED1 V01Y2015 09IVM019.

[15] Floater, M. S., Hormann, K., Surface Parameterization: a Tutorial and Survey. Advances in Multiresolution for Geometric Modelling, pp. 157-186.

https://doi.org/10.1007/3-540-26808-19

[16] Dodgson, N., Floater, M. S., Sabin, M., Advances in multiresolution for geometric modelling. Springer, Berlin, Heidelberg, 2005.

[17] Barrile, V., Fotia A., Bilotta G., De Carlo, D., Integration of geomatics methodologies and creation of a cultural heritage app using augmented reality. Virtual Archaeology Review, Vol.10, No.20, 2019, pp. 40-51. https://doi.org/10.4995/var.2019.10361
[18] Cowan, B., Kapralos, B., A Survey of Frameworks and Game Engines for Serious Game Development, IEEE 14th International Conference on Advanced Learning Technologies, 2014, pp. 662-664. 10.1109/ICALT.2014.194

[19] Hayden S., VR Tour App 'MasterWorks' Uses Photogrammetry to Bring You to 4 Fully Explorable Heritage Sites, Road to VR, 2018. https://www.roadtovr.com/masterworks-uses photogrammetry-take-guided-vr-tour-4-fully explorable-heritage-sites/

[20] Bruno, F., Lagudi, A., Barbieri, L., Cozza, M., Cozza, A., Peluso, R., Davidde Petriaggi, B., Petriaggi, R., Rizvic, S., and Skarlatos, D., Virtual tour in the sunken "villa con ingresso a protiro" within the underwater archaeological park of Baiae. In. Arch. Photogramm. Remote Sens. Spatial Inf. Sci., Vol.XLII-2/W10, 2019, pp. $45-51$.

https://doi.org/10.5194/isprs-archives-XLII-2 W10-45-2019

[21] Manferdini, A. M., Gasperoni, S., Guidi, F., \& Marchesi, M., Unveiling Damnatio Memoriae. The use of $3 \mathrm{D}$ digital technologies for the virtual reconstruction of archaeological finds and artefacts, Virtual Archaeology Review, Vol.7, No.15, 2016, pp. 9-17. https://doi.org/10.4995/var.2016.5871.

[22] Esclapés, J., Tejerina, D., Esquembre, M. A., \& Bolufer, J., Methodological proposal to generate interactive virtual walkthrough, Virtual Archaeology Review, Vol.4, No.9, 2013, pp. 212-222. https://doi.org/10.4995/var.2013.4276.

[23] Caro, J.L., Hansen, S., From photogrammetry to the dissemination of archaeological heritage using game engines: Menga case study, Virtual Archaeology Review, Vol.6, No.12, 2015, pp. 58-68. https://doi.org/10.4995/var.2015.4159

[24] Previtali, M., \& Valente, R., Archaeological documentation and data sharing: digital surveying and open data approach applied to archaeological fieldworks. Virtual Archaeology Review, Vol.10, No.20, 2019, pp. 17-27. https://doi.org/10.4995/var.2019.10377

[25] Ferrari, I., \& Quarta, A., The Roman pier of San Cataldo: from archaeological data to 3D reconstruction. Virtual Archaeology Review, Vol.10, No.20, 2019, pp. 28-39. https://doi.org/10.4995/var.2019.7957. 
[26] Zulkifli, Boy Syamsul Bakhri, Muhammad Yusuf, An Analysis of Citizens' Understanding on Sharia Tourism in Pekanbaru City, Indonesia WSEAS Transactions on Business and Economics, pp. 205-214, Volume 16, 2019 ,

[27] Chun-Chu Liu, Jen-Shou Kao A Study of Stay Motivation, Service Quality, and Satisfaction of B\&B Tourists - A Case Study of Kenting in Taiwan WSEAS Transactions on Business and Economics, pp. 171-179, Volume 15, 2018,

\section{Creative Commons Attribution License 4.0}

(Attribution 4.0 International, CC BY 4.0)

This article is published under the terms of the Creative Commons Attribution License 4.0

https://creativecommons.org/licenses/by/4.0/deed .en_US 